\title{
High-Throughput Characterization of Porous Materials Using Graphics Processing Units
}

\author{
Jihan Kim,,$^{* \dagger}$ Richard L. Martin,,${ }^{\ddagger}$ Oliver Rübel, ${ }^{\ddagger}$ Maciej Haranczyk, ${ }^{\ddagger}$ and Berend Smit ${ }^{\text {II }}$ \\ ${ }^{\dagger}$ Material Sciences Division, Lawrence Berkeley National Laboratory, Berkeley, California 94720, United States \\ ‡Computational Research Division, Lawrence Berkeley National Laboratory, Berkeley, California 94720, United States \\ ${ }^{\mathbb{T}}$ Departments of Chemical and Biomolecular Engineering and Chemistry, University of California, Berkeley, California 94720, United \\ States
}

ABSTRACT: We have developed a high-throughput graphics processing unit (GPU) code that can characterize a large database of crystalline porous materials. In our algorithm, the GPU is utilized to accelerate energy grid calculations, where the grid values represent interactions (i.e., Lennard-Jones + Coulomb potentials) between gas molecules (i.e., $\mathrm{CH}_{4}$ and $\mathrm{CO}_{2}$ ) and materials' framework atoms. Using a parallel flood fill central processing unit (CPU) algorithm, inaccessible regions inside the framework structures are identified and blocked, based on their energy profiles. Finally, we compute the Henry coefficients and heats of adsorption through statistical Widom insertion Monte Carlo moves in the domain restricted to the accessible space. The code offers significant speedup over a single core CPU code and allows us to characterize a set of porous materials at least an order of magnitude larger than those considered in earlier studies. For structures selected from such a prescreening algorithm, full adsorption isotherms can be calculated by conducting multiple Grand Canonical Monte Carlo (GCMC) simulations concurrently within the GPU.

\section{INTRODUCTION}

Porous materials, such as zeolites and metal-organic frameworks (MOFs), have been exploited in many current technologies and are considered to be a very important class of materials for many new industrial applications. For example, zeolites are commonly used as chemical catalysts, in particular as cracking catalysts in oil refinement, membranes for separations, and water softeners. ${ }^{1-4}$ In addition, there is an increasing interest in utilizing zeolites as membranes or adsorbents for $\mathrm{CO}_{2}$ capture applications. ${ }^{5-8}$ Other materials, such as $\mathrm{MOFs}^{9,10}$ and their subfamily of zeolitic imidazolate frameworks (ZIFs), ${ }^{11}$ have enormous potential for gas separations and storage as well. ${ }^{12,13}$

A key factor that determines the utility of any nanoporous material is its optimal pore topology along with the chemical composition for given conditions in a particular application. There are $\sim 190$ known unique zeolite frameworks ${ }^{14}$ in more than 1400 zeolite crystals of various chemical composition and geometry. ${ }^{15}$ However, these experimentally known zeolites constitute only a very small fraction of more than 2.7 million structures that are feasible on theoretical grounds. ${ }^{16,17}$ Of these, between 300000 and 600000 are predicted to be thermodynamically accessible as aluminosilicates, with the remainder also potentially accessible via elemental substitution. ${ }^{18,19}$ All of the zeolite structures in this research work are comprised of silicon and oxygen atoms, making these materials much more simple in terms of their chemical composition than ZIFs or MOFs. The chemical composition of zeolite structures can be altered by replacing some of the silicon atoms with aluminum (or other) atoms, and then adding cations (e.g., $\mathrm{Na}^{+}$) to impose charge neutrality. Changing the chemical makeup of zeolite materials in this way further increases the number of possible structures. Structure sets of similar or even greater size are expected for other nanoporous materials such as ZIFs, ${ }^{7}$ which offer greater flexibility in the choice of building blocks.

In an attempt to identify optimal materials for various applications, such as gas separations, ${ }^{5-7}$ researchers have started to screen large databases of porous materials. Molecular simulation techniques, such as the Grand Canonical Monte Carlo (GCMC) method, are often used in numerical simulations to accurately predict properties of materials and their guest-adsorption characteristics are expressed as an experimentally verified adsorption isotherm..$^{20-22}$ However, the computational cost of molecular simulations is high, significantly limiting the number of structures that can be analyzed. To avoid the high computational costs, most of the screening strategies rely on a thorough prescreening of materials, which filters out structures based on easily obtainable structural properties, such as pore diameters and framework energy. As an example, Haldouplis et al. screened 250000 zeolites, while only about 8000 were characterized using molecular simulations. ${ }^{6}$ Nonetheless, it is important to note developments in algorithms that allow high-throughput characterization of porous materials via generation of structural parameters used for prescreening ${ }^{5,23-25}$ or database sampling approaches. $^{26}$

The importance of these screening strategies motivated us to approach this task from a high-performance computing point of view and utilize fast molecular simulation techniques that allow us to characterize a very large set of porous materials-more than an order of magnitude larger than what has been reported previously by other researchers. In addition, we addressed a practical complication limiting the characterization of large sets

Received: November 5, 2011

Published: March 16, 2012 
of materials, which is the determination of whether pores in a material are accessible. This step typically involves visual inspection, ${ }^{35}$ which becomes cumbersome with large numbers of structures. Our approach integrates an automatic analysis of topology of the materials' void space, and accordingly, the simulation domain can be defined within the accessible void space of a material and this domain reflects the space available to molecules in experiments.

In order to process large sets of materials within a reasonable time, our molecular simulation tool utilizes graphical processing units (GPUs) to efficiently conduct parallel calculations. GPUs are hardware accelerators that were initially developed to accelerate graphics-related tasks. With the advent of NVIDIA's CUDA (compute unified device architecture) and subsequent development of the CUDA software interface, general purpose GPU (GPGPU) programming has become more prevalent and commonplace in the scientific community. ${ }^{27}$ Unlike conventional CPUs, GPUs have many more transistors devoted to data processing and, as such, can provide significant performance improvement in computational problems that can be easily mapped onto its multithreaded hardware. In the context of molecular simulations, GPGPU computing has been mainly used to accelerate molecular dynamics (MD) and Monte Carlo (MC) simulations. ${ }^{28-31}$ Our molecular simulation GPU code takes advantage of the fact that the computationally intensive bottleneck routines can easily be mapped into a SIMD (same instruction multiple data) format, making it ideal to port the code from the CPU to the GPU. Although this article focuses on GPU simulation results of the zeolite structures, the code can be easily extended to process other important classes of porous materials, such as MOFs or ZIFs, and can accelerate characterization in those materials as well. As such, the techniques described in this work can be generalized well to many other systems.

The manuscript is organized as follows. In Section 2, we discuss the algorithmic details of our hybrid GPU + CPU characterization/screening code. In Section 3, we analyze the performance of our implementation and present results obtained using our GPU code. In Section 4, we summarize the important findings in our work and discuss avenues for future work.

\section{ALGORITHM FOR CHARACTERIZING POROUS MATERIALS}

In this work, we focus on $\mathrm{CO}_{2}$ and $\mathrm{CH}_{4}$ gas molecules, because they comprise representative examples for the behavior of molecules with, respectively, partial atomic charges and no charges. However, the techniques described in this work can readily extend to other gas molecules such as $\mathrm{N}_{2}, \mathrm{He}$, and $\mathrm{H}_{2} \mathrm{O}$ as well. The zeolite framework is assumed to be rigid, which is a reasonable approximation ${ }^{3}$ and, as such, only the gasframework and the gas-gas interactions are considered. As a means to characterize the zeolite structures, we compute the Henry coefficient $\left(K_{\mathrm{H}}\right)$ and the heat of adsorption $\left(\Delta h_{i}\right)$ values of $\mathrm{CO}_{2}$ and $\mathrm{CH}_{4}$. These quantities characterize adsorption of the gas molecules in porous materials. $K_{\mathrm{H}}$ is a basic constant that relates the equilibrium between the gas and the adsorbed phase $\left(\rho=K_{\mathrm{H}} P\right)$ and, hence, describes adsorption in the verylow-pressure regime. In the context of $\mathrm{CO}_{2}$ capture, an ideal material would exhibit a large $\mathrm{CO}_{2} K_{\mathrm{H}}$ value, compared to the $K_{\mathrm{H}}$ values of other flue gases, resulting in high selectivity for $\mathrm{CO}_{2}$. For carbon capture of flue gases, the pressure values are relatively low and for most systems, $K_{\mathrm{H}}$ serves as an important quantity to characterize large material databases. $K_{\mathrm{H}}$ and $\Delta h_{i}$ can be computed from Monte Carlo simulations:

$$
K_{\mathrm{H}}=\beta\left\langle\exp \left(-\beta U_{\text {ins }}\right)\right\rangle_{\text {test }}
$$

and

$$
\Delta h_{\mathrm{i}}=\frac{\left\langle U_{\text {ins }} \exp \left(-\beta U_{\text {ins }}\right)\right\rangle}{\left\langle\exp \left(-\beta U_{\text {ins }}\right)\right\rangle}
$$

with $\beta=1 /\left(k_{\mathrm{B}} T\right)$ with $k_{\mathrm{B}}$ representing the Boltzmann constant and $T$ indicating the temperature of the system. $T$ is fixed at $300 \mathrm{~K}$ in all simulations reported in this work. $U_{\text {ins }}$ represents the test particle energy of the gas molecule at a random position in the material. $\langle\cdot\rangle_{\text {test }}$ indicates that, in the ensemble average, the test particle does contribute to the energy of the system. Upon taking a sufficiently large number of Monte Carlo Widom insertions, which consist of randomized insertions of the gas molecules in the simulated volume, the $K_{\mathrm{H}}$ and $\Delta h_{i}$ values of the gas molecules can be computed with a great deal of accuracy.

The $K_{\mathrm{H}}$ and $\Delta h_{i}$ computational algorithms for a given porous material consist of the following three important steps:

(1) Construct an energy grid that stores the energy values of the test gas molecule at discrete positions of the structure's unit cell (to be discussed in Section 2.1),

(2) Automatically identify inaccessible regions within the structure, utilizing the energy grid values from the previous calculation (to be discussed in Section 2.2), and

(3) Conduct Widom insertion Monte Carlo moves to compute the Henry coefficient $K_{\mathrm{H}}$ and the heats of adsorption $\Delta h_{i}$ of the gas molecule (to be discussed in Section 2.3).

This algorithm is utilized to characterize all materials in the theoretical zeolite database. We describe the algorithm outlined above in detail in the subsequent subsections. In Section 2.4, we briefly describe the GCMC algorithm used to compute the adsorption isotherms inside the GPU, which can provide adsorption properties of the materials in higher-pressure regimes.

2.1. Energy Grid Construction. All of the zeolite materials in our simulations are crystalline structures, and, accordingly, the adsorption properties of each of these materials can be accurately characterized by examining a small number of unit cells and imposing a periodic boundary condition. Inside the numerical domain of a single unit cell, we construct a threedimensional energy grid for each of the zeolite structures. The grid points of the energy grid each represent the sum of the Lennard-Jones and the Coulomb potentials between the gas molecule and all of the framework atoms that make relevant contribution to the interaction. The spacing of the energy grid is fixed to be $0.1 \AA$ along all three spatial dimensions. The Lennard-Jones potential and the Coulomb potentials are defined as follows:

$$
U_{\mathrm{LJ}}(r)=4 \varepsilon\left[\left(\frac{\sigma}{r}\right)^{12}-\left(\frac{\sigma}{r}\right)^{6}\right]
$$

and

$$
U_{\text {coul }}(r)=\frac{q_{i} q_{j}}{4 \pi \varepsilon_{0} r}
$$

Here, $r$ represents the distance between two particles, $\varepsilon$ indicates the depth of the potential well, $\sigma$ represents the 
effective core size of the particles with the potential well located at $2^{1 / 6} \sigma, q_{i}$ and $q_{j}$ indicate the charges of the two particles, and $\varepsilon_{0}$ represents the material permittivity. For all pairwise interactions, a Lennard-Jones cutoff radius $R_{\mathrm{C}}=12.0 \AA$ is imposed, such that the interaction is shifted to zero for $r>R_{\mathrm{C}}$ by subtraction of $U_{\mathrm{LJ}}\left(R_{\mathrm{C}}\right)$ from $U_{\mathrm{LJ}}(r)$ for all $r \leq R_{\mathrm{C}}$. In our code, the long-range Coulomb interactions between the charged particles are approximated by the Ewald summation method. The work to compute the periodic Coulomb potentials is divided into summations in both real and Fourier components to accelerate convergence. All of the force fields used in this work come from the research of Garcia-Perez et al. $^{32}$ and Dubbeldam et al., ${ }^{33}$ which have been shown to reproduce the experimental adsorption for various zeolite structures.

Given that a very large number of energy grid points must be computed (over $10^{8}$ for most zeolite structures), GPU hardware architecture can be efficiently utilized by employing thousands of CUDA threads to concurrently compute the energy values at these points in parallel. In our algorithm, the total number of CUDA threads is set to be equal to the total number of energy grid points, creating a one-to-one mapping between threads and grid points. Accordingly, each CUDA thread computes the interaction between the gas molecule at the specified grid point and all the framework atoms. The number of framework atoms $\left(N_{\text {tot }}\right)$ is, in most zeolites, small enough $\left(N_{\text {tot }}<2000\right.$ atoms $)$ such that all data required fits into the $64 \mathrm{kB}$ constant memory of the Tesla C2050 Fermi cards used, minimizing the number of GPU DRAM transactions. For $N_{\text {tot }}>2000$, the data containing the framework atom positions is stored in the slower GPU DRAM, which leads to a decrease in performance. In practice, this performance deterioration is small and has no major impact on our experiments. Also, in the hypothetical zeolite database, the number of structures with $N_{\text {tot }}>2000$ is relatively small and, thus, the overall wall time to process an entire database is not affected significantly by the reduced memory bandwidth. Since the constant memory bandwidth is greater than the shared/L1 cache memory and given that we only require read operations from the framework atoms, the decision was made to choose constant memory over other fast memory available in the GPU. For a linear molecule such as $\mathrm{CO}_{2}$, we compute separate carbon and oxygen grids for the Lennard-Jones interaction while computing only one Coulomb grid (e.g., carbon atom Coulomb grid). The Coulomb interaction values for the second atom (i.e., oxygen) can be obtained by multiplying the grid point values of the carbon Coulomb grid by a prefactor corresponding to the ratio of the charges of the two atoms. This strategy does not only reduce computation time, but it also reduces the amount of GPU DRAM required; this is important given the 3GB DRAM constraint of the Tesla C2050 cards.

In order to determine inaccessible regions within the unit cell of the structure (described in Section 2.2), an additional energy grid is required. This additional grid is constructed to encode the total energy of the gas molecule at each of the grid points, and accordingly approximately maps to the occupation probability of the gas molecule at that position (i.e., a higher total energy corresponds to a lower probability of occupancy). For molecules such as $\mathrm{CH}_{4}$, which are modeled as point particles, this total energy grid is equivalent to the individual energy grid computed in the energy grid construction routine. However, for linear molecules, such as $\mathrm{CO}_{2}$ and $\mathrm{N}_{2}$, or nonlinear molecules, such as $\mathrm{H}_{2} \mathrm{O}$, the total energy values must be obtained separately from the individual energy grids computed by the grid construction routine. We obtain approximate values of energies of these molecules and store the result in a single grid point by conducting a large number of test rotation moves about the grid point and computing its average energy. In $\mathrm{CO}_{2}$, for example, the carbon atom is positioned to coincide with each grid point in turn, and $N_{\text {rot }}=$ 100 random rotations are conducted on the two oxygen atoms about that point. Because rotations can be conducted independently and in parallel, the test rotation routine maps well to the GPU. We utilize the CUDA CURAND library to generate the random numbers that determine the gas molecule orientations. The energy value for the carbon atom is sampled directly from the Lennard-Jones and the Coulomb energy grids, whereas the energy values for the two randomized oxygen atoms are obtained using linear interpolation functions from the energy grid values. The trilinear interpolation function samples eight nearest-neighbor points in the energy grid from the rectangular voxel that encompasses the sampled point. In cases where the identification of inaccessible regions within a structure is unnecessary, the test rotation routine can be skipped in order to improve computational performance.

2.2. Pocket Blocking Algorithm. In some materials, the arrangement of atoms in the structure is such that there exist regions of space that a guest molecule could occupy, but which it cannot access, because of the positions of the surrounding atoms. These regions constitute "inaccessible pockets" of void space, and they are contrasted with accessible regions of space (i.e., "channels"). In computer calculations, it is critical to account for these positions such that they are not considered in, for example, the calculation of guest-accessible volumes or surface areas, or the prediction of adsorption properties using molecular simulation techniques. ${ }^{34,35}$ It is typical to detect inaccessible pockets through visual inspection of the so-called pore landscapes, and subsequently to block them with exclusion spheres. ${ }^{36}$ However, it is not practical to perform a visualization-based analysis on the very large quantity of materials with which we are concerned. Accordingly, we have recently developed algorithms for the automatic segmentation of void space (into accessible and inaccessible regions) and the exclusion of pockets. ${ }^{37}$

The original algorithms ${ }^{37}$ relied on partial differential equation (PDE)-based front propagation techniques, by which the grid representing the guest-accessible positions (or, in the case of complex nonspherical probes, guest accessible orientations at each position ${ }^{38}$ ) can be segmented. There are numerous advantages to a PDE-based segmentation algorithm, including the approximation of paths of least resistance between certain positions within the structure, which can give insights regarding diffusion. However, for the purposes of highthroughput characterization-on the order of hundreds of thousands of structures or more-the additional information obtained by solving the PDE is generally not of critical importance. Therefore, in order to accelerate this process, we perform a more simplistic segmentation using a parallel flood fill (also known as seed fill, boundary fill, or bucket fill) algorithm described in ref 39. Flood fill is a recursive algorithm to determine the bounds of a connected region, and therefore, similar to PDE-based methods, can segment a grid into distinct, connected regions of guest-accessible space. Following this process, regions that connect across the periodic boundary are detected and merged. In our parallel implementation, each CPU thread is assigned a separate subdomain of the unit cell, 
upon which it performs segmentation using flood fill. Connections across subdomain boundaries are considered in the same manner as those that cross the periodic boundary of the cell. We then proceed to identify and block inaccessible regions, as discussed in the following paragraphs.

In this study, we represent the material with a threedimensional energy grid. The energy terms calculated at each discrete grid point can be interpreted as conveying the probability of the guest molecule occupying that position in the form of the Boltzmann factor, $\exp \left(-\beta E_{i}\right)$ with $E_{i}$ representing the energy of the $i$ th grid point. We interpret this grid in a binary fashion, as containing grid points that can or cannot be occupied by the guest molecule in the time frame of our application. We set the following:

$$
\text { if }\left(E_{i}<(p T)\right) \rightarrow \text { accessible; else } \rightarrow \text { inaccessible }
$$

where $T$ is the temperature, and $p$ relates to the probability of the position being occupied. At long time scales (for instance, in geologic applications), high barriers can be overcome, and so $p$ can take a high value; however, for our carbon capture gas separation application, we set $p=15$, such that a point is accessible if $\exp \left(-E_{i}\right)<\exp (-15 T)$. This number was chosen to be large enough such that, in a typical zeolite crystal structure, these forbidden regions are considered to be diffusively inaccessible on an experimental time scale. From this binary interpretation of the energy grid, the material's unit cell is segmented into disconnected, nonperiodic regions of void space using parallel flood fill. Each of these distinct regions is then analyzed to determine whether it forms a channel or an inaccessible pocket. We examine the positions where each region reaches a face of the unit cell, and inspect their periodic neighbors for accessibility, connecting these regions; the boundaries between each CPU thread's flood fill domain are inspected in the same manner. Using this method, we classify regions as "channels" if they constitute a loop through the void space; otherwise, we classify them as "pockets" (see Figure 1).

As discussed previously, it is important to exclude inaccessible pockets prior to performing techniques such as MC sampling in order to avoid false contributions of the energy term within inaccessible regions to the measured behavior of the overall system. We have devised two techniques for the

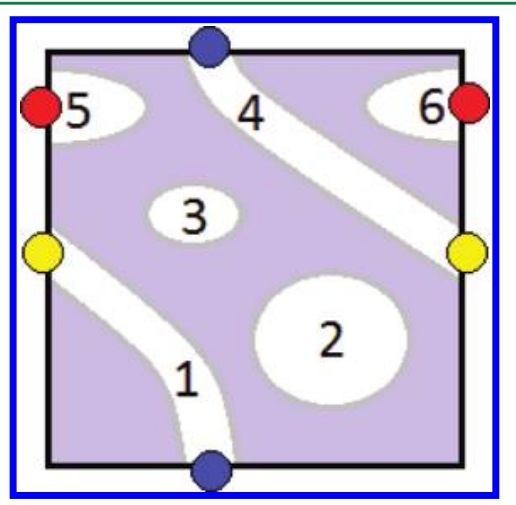

Figure 1. Two-dimensional illustration of the pocket blocking technique. Having segmented the energy grid into distinct regions of occupiable space, we examine the positions where regions touch the periodic boundary. Hence, we merge regions 1 and 4 and find that they form a channel; regions 2 and 3 are pockets, since they do not reach the boundary; and regions 5 and 6 are merged to form a pocket which crosses the periodic boundary. exclusion of these inaccessible regions. The first approach is to generate a set of exclusion spheres that span each pocket, without interfering with other regions. The algorithm for generating these spheres is described in ref 37 . In the subsequent $\mathrm{MC}$ step, moves that are within an exclusion sphere are rejected. The obtained set of blocking spheres can be visualized or used in other molecular simulation packages. The second approach, which is utilized in our high-throughput analysis, is simply to mark grid points that are within inaccessible space by setting their energy-grid values to be prohibitively large. This saves time, by bypassing the generation of blocking spheres, and in the Widom insertion step the high values at these points are sufficient to identify the inaccessible space. This constitutes a further performance improvement, with respect to our earlier approaches. ${ }^{37,39}$ Both of these techniques are implemented in our tool as multicore CPU functions, wherein each thread works independently to exclude an individual pocket.

2.3. Widom Insertion Monte Carlo. Utilizing the energy grids, the $K_{\mathrm{H}}$ and the $\Delta h_{\mathrm{i}}$ of gas molecules inside porous materials can be calculated via Widom insertion Monte Carlo (MC) moves. Similar to the test rotation routine, Widom insertion is conducted inside the GPU, again utilizing the CUDA CURAND library for random number generation. For $\mathrm{CH}_{4}$, the insertion algorithm entails choosing random particle positions uniformly sampled from the entire simulation box and repeating this process while sampling the Lennard-Jones potential values via interpolation using values from the energy grid. For $\mathrm{CO}_{2}$, the carbon atom is randomly placed uniformly sampled from the entire simulation volume, similar to the case of $\mathrm{CH}_{4}$, while both the Lennard-Jones and the Coulomb interaction energies of the carbon atom are sampled from the energy grids via linear interpolation functions. In zeolite structures that have nonorthogonal unit cells, the randomly generated position value might fall outside of the unit cell, which causes the point to be rejected without making contribution to the MC statistics. Also, if the position of the carbon atom falls inside the inaccessible region, the sampled energy value would be very high due to the high energy values set in the CPU pocket blocking routine. The energy value is set high enough (i.e., 1 million $k_{\mathrm{B}} T$ ) such that the entire $\mathrm{CO}_{2}$ molecule would possess large energy, regardless of the values sampled from the other two oxygen atoms. The same holds true in case that any of the atoms falls inside the inaccessible region. Once the insertion of the carbon atom is finished, the algorithm proceeds to insert the first oxygen atom by randomly sampling from a surface of a sphere with a radius equal to the bond length of $\mathrm{CO}_{2}$ (i.e., $1.16 \AA$ ). If the oxygen atom falls outside of the unit cell, then the periodic boundary condition is used to move the position of the atom back inside the unit cell via appropriate displacements. Finally, the second oxygen atom is placed in a position such that all three atomic positions are collinear to each other. The placement of the final atom in the linear molecule does not require generation of random numbers, due to the fact that there are zero degrees of freedom. Inside the code, variables that store the total energy and the Boltzmann factors are updated at each iteration of the Widom insertion MC cycles. For the GPU thread configurations, $16 \times 14=224$ CUDA blocks are generated with a block size of 64 in the CUDA kernel for the Widom insertions. Each of the CUDA threads conduct 1000 independent Widom MC cycles, resulting in a total of $\sim 14$ million insertion moves. Unless the CUDA occupancy is set to be too low, the block size 
and the number of blocks can be changed easily without disrupting the code's functionality or performance.

Rather than computing the adsorption properties for the entire structure, the code can also calculate the local $K_{\mathrm{H}}$ and $\Delta h_{i}$ values of the gas molecule within a specified subset region of our simulation volume. This capability allows us to focus on different regions within the porous material to obtain a better understanding on the local adsorption properties of the regions of interest. In the simulation code, the subset region is described by the union of several spheres at different positions and various radii. In the local $K_{\mathrm{H}}$ and $\Delta h_{i}$ calculations, the Widom insertion algorithm first checks to see whether the $\mathrm{CO}_{2}$ molecule is inside any of the spheres that describe the local region and rejects any other insertion moves. Accordingly, in the limiting case where the spheres cover the entire simulation volume, the problem simply reduces back to the original Widom insertion algorithm. If the total volume of the spheres is much smaller than the volume of the unit cell, most of the Widom insertion moves will be rejected, because the probability of sampling the location regions will be small. Having a large number of rejected moves can slow the code significantly, because of (i) the warp divergence that occurs within the GPU threads and (ii) the high cost of generating random numbers inside the GPU. However, because the local structural properties analysis is something with which we are interested for only a small number of structures at this point, we have yet to optimize this part of the code. In the Results section, we elaborate on some of the findings that come from sampling local regions of the zeolite structures.

2.4. Adsorption Isotherm Calculations. Grand Canonical Monte Carlo (GCMC) simulations are utilized to compute adsorption isotherms. Given the relatively small number of gas particles found within zeolite structures, there exists an insufficient amount of work that can be efficiently parallelized via thousands of threads within the GPU. In order to circumvent this issue, we propose a parallelization strategy in which multiple Monte Carlo (MC) simulations are conducted in parallel inside the GPU. ${ }^{28}$ Specifically, the number of different pressure values for the GCMC simulation is set to be 14 , which is equal to the number of streaming multiprocessors (SMs) found in the Tesla C2050 card, and each SM is responsible for conducting a single GCMC simulation. The threads within the CUDA blocks can work together to parallelize the different pairwise interaction contributions and a reduction kernel can be used to sum up the contributions from each of the individual threads. The energy grids computed in the previous steps are still utilized to remove the need to explicitly compute the gas-host interactions at each step of the MC cycles. Furthermore, there can be an additional speedup by tabulating the Fourier components of the gas-gas Ewald summation interactions in another energy grid to circumvent the need to iterate over all of the $k$ vectors for each of the pairwise interactions during the $\mathrm{MC}$ cycles. ${ }^{40}$ Similar to the gas-host energy grid, the linear interpolation functions are utilized to estimate the gas-gas interaction. Within the periodic boundary conditions, the Fourier components are only a function of the vector distance between two particles and thus we do not lose much accuracy upon utilizing the tabular grid.

\section{RESULTS}

In the simulation results, we initially focus our attention on the experimentally verified 187 IZA zeolite structures and later extend our analysis to a much larger hypothetical zeolite database. All of the numerical simulations were performed on the Dirac and Carver clusters, located at the National Energy Research Scientific Computing Center (NERSC). Dirac is a testbed GPU cluster consisting of 48 nodes (44 Tesla C2050 Fermi GPU cards and 4 Tesla 1060 GPU cards). Each node contains two Quad core Intel Nehalem $55302.4 \mathrm{GHz}$ processors with an $8 \mathrm{MB}$ cache, $5.86 \mathrm{GT} / \mathrm{s}$ QPI. The Fermi GPUs have 448 CUDA cores, a PCIe x16 Gen2 system interface, and 3 GB of GDDR5 memory-where a portion of the memory $(12.5 \%)$ is dedicated to error correction code (ECC) bits-yielding $2.625 \mathrm{~GB}$ of user available memory. According to the NVidia's Tesla C2050 specifications, the double (single)-precision floating point performance number peaks at 515 GFLOPS (1.03 TFLOPS), while the memory bandwidth is indicated to be $144 \mathrm{~GB} / \mathrm{s}$. In order to utilize as many GPUs as possible on the Dirac cluster, we use a simple MPI+CUDA multi-GPU version of the code, which supports static as well as dynamic scheduling of the material structures onto the MPI tasks for processing. The CPU simulations were performed on the Carver cluster, which consists of 800 Intel Nehalem 2.67 GHz quad-core processors with 24 GB DDR3 $1333 \mathrm{MHz}$ memory. Finally, we used the CUDA Toolkit 3.2, the CURAND Library for random number generations, and gcc 4.4.2 compiler with full optimizations in all of our simulations.

3.1. Performance Analysis. 3.1.1. Overall Performance. Figure 2 shows the timing results for the $\mathrm{CH}_{4}$ and $\mathrm{CO}_{2} \mathrm{~K}_{\mathrm{H}}$ and

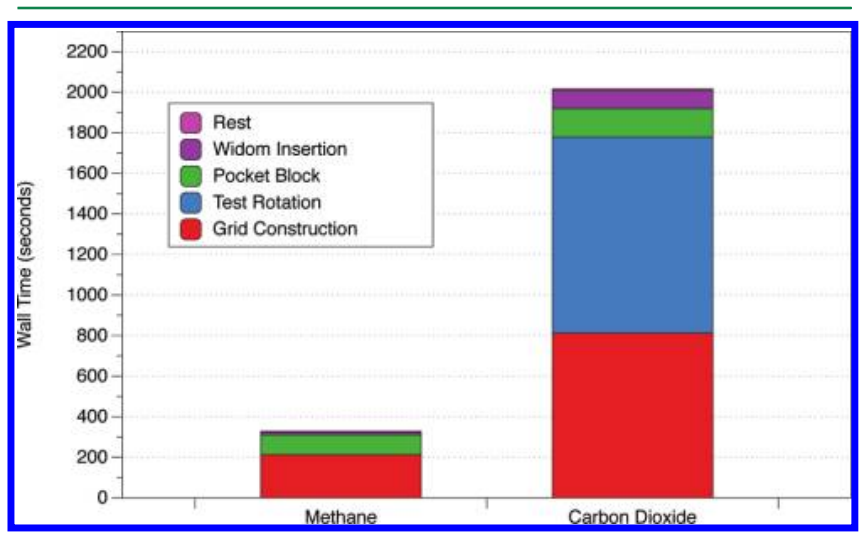

Figure 2. Distribution of wall time for 187 IZA structures with methane and carbon dioxide gas molecules. The energy grid size is set to be $0.1 \AA$, and the number of test rotations about the grid points is set at 100 , and roughly 10 million Widom insertion Monte Carlo moves. The total computational time is divided by the major routines that comprise the simulation code: (1) GPU energy grid construction, (2) GPU test rotation, (3) CPU pocket blocking, and (4) GPU Widom insertion moves.

$\Delta h_{i}$ calculations for the IZA structures. Given that the two quantities are computed simultaneously in the Widom insertion routine, we focus only on the $K_{\mathrm{H}}$ results in our performance analysis.

The total computational wall time for all of the $187 \mathrm{IZA} \mathrm{CH}_{4}$ and $\mathrm{CO}_{2}$ simulations are measured to be 327.59 and $2016.28 \mathrm{~s}$, respectively. Accordingly, the GPU code requires $\sim 1.74 \mathrm{~s}$ $\left(\mathrm{CH}_{4}\right)$ and $\sim 10.72 \mathrm{~s}\left(\mathrm{CO}_{2}\right)$ to compute a single value of $K_{\mathrm{H}}$ and $\Delta h_{i}$, making the $\mathrm{CH}_{4}$ calculations $\sim 6.2$ times faster than the $\mathrm{CO}_{2}$ calculations. In general, the $\mathrm{CO}_{2}$ calculations require longer wall time, largely due to (a) the presence of charge in the carbon and the oxygen atoms of the $\mathrm{CO}_{2}$ molecules, necessitating the expensive Ewald summation computations, and (b) invoking calls to the test rotation routine. Breaking 
down the performance of individual routines in further detail, we see that the $\mathrm{CH}_{4}\left(\mathrm{CO}_{2}\right)$ energy grid construction routine takes $212.52(813.15)$ s, making the $\mathrm{CO}_{2}$ calculations 3.83 times more expensive, because of the presence of Coulomb interaction terms. The number of terms in the Fourier space calculations for the Ewald summation scales with $\mathrm{O}\left(k_{\max }^{3}\right)$, with $k_{\max }$ representing the maximum number of $k$-vectors. We can accelerate the Fourier space calculations inside the GPU by nearly 2 -fold by replacing the sine and cosine terms with the sincos function for terms located in the innermost loop of the nested $k$-vector. In the $\mathrm{CO}_{2}$ calculations, $47.9 \%$ of the wall time is spent in the test rotation routine, as opposed to $0 \%$ for $\mathrm{CH}_{4}$, since the rotations are only required for multiatom, linear, and nonlinear molecules. Later in the paper, we explore the performance impact of varying the number of test rotations in this routine. The amount of time spent in pocket blocking for $\mathrm{CH}_{4}\left(\mathrm{CO}_{2}\right)$ is 97.11 (139.93) s, making the performance for the different guest molecules comparable. The additional Coulomb interaction term in the $\mathrm{CO}_{2}$ calculations often produce more-complicated energy profiles in the simulation box, which can increase the time spent in the flood fill step. Finally, the wall time spent in the Widom insertions is proportionally small, compared to the overall wall time in both $\mathrm{CH}_{4}(12.51 \mathrm{~s})$ and $\mathrm{CO}_{2}(87.91 \mathrm{~s})$ calculations. This is not surprising, given that computational intensity is low in the Widom insertion moves, because the routine involves mostly reading precomputed energy grid values from an array stored inside the GPU. The performance numbers for the Widom insertions indicate that the wall time is 7.02 times larger in the case of $\mathrm{CO}_{2}$ compared to $\mathrm{CH}_{4}$, which can be explained by the following. $\mathrm{CO}_{2}$ calculations require two random and one nonrandom insertion, as well as interpolation of values from both the Lennard-Jones and the Coulomb grid, to obtain an energy value for a single configuration. On the other hand, the $\mathrm{CH}_{4}$ molecule entails one random insertion and one interpolation from the Lennard-Jones grid for a single configuration. The number of MC cycles for the Widom insertion routine can be changed depending on the level of accuracy desired for the $K_{\mathrm{H}}$ and $\Delta h_{i}$ results, which can affect the proportional wall time spent in this routine as its wall time scales linearly, with respect to the number of cycles.

3.1.2. Numerical Accuracy: Energy Grid Size. Next, we analyze the relationship between the energy grid size and code performance. In general, the computational wall time for the $K_{\mathrm{H}}$ and $\Delta h_{i}$ calculations can be reduced by increasing the mesh size of the energy grid. In practice, the grid size should be chosen to be small enough to ensure numerical accuracy of the $K_{\mathrm{H}}$ and $\Delta h_{i}$ calculations performed using the energy grid. In earlier work on GPU waste recycling MC, ${ }^{28}$ we have demonstrated that, in the zeolite MFI structure for $\mathrm{CH}_{4}$ molecules, utilizing the energy grid provides average energy values within $0.05 \%$ of those obtained from direct Lennard-Jones potentials without the grid, providing good justification of using the grid.

In Figure 3, the mean values of $\mathrm{CO}_{2} \mathrm{~K}_{\mathrm{H}}$ of five IZA structures (i.e., MAR, FAR, LTA, FAU, and MTN) are plotted for different mesh sizes $\left(d_{\mathrm{m}}=0.075,0.10,0.15,0.20,0.25\right.$, and 0.30 $\AA$ ). These 5 structures are part of a blocking set of 20 IZA, which includes zeolite structures that possess inaccessible regions for the $\mathrm{CO}_{2}$ molecules; this set comprises $\sim 11 \%$ (20/ 187 ) of the entire IZA database. It is not necessary to plot the curves for all the structures in the blocking set, because these 5 are sufficient to show the general trends for all blocking structures, with respect to changes in the grid size. As can be

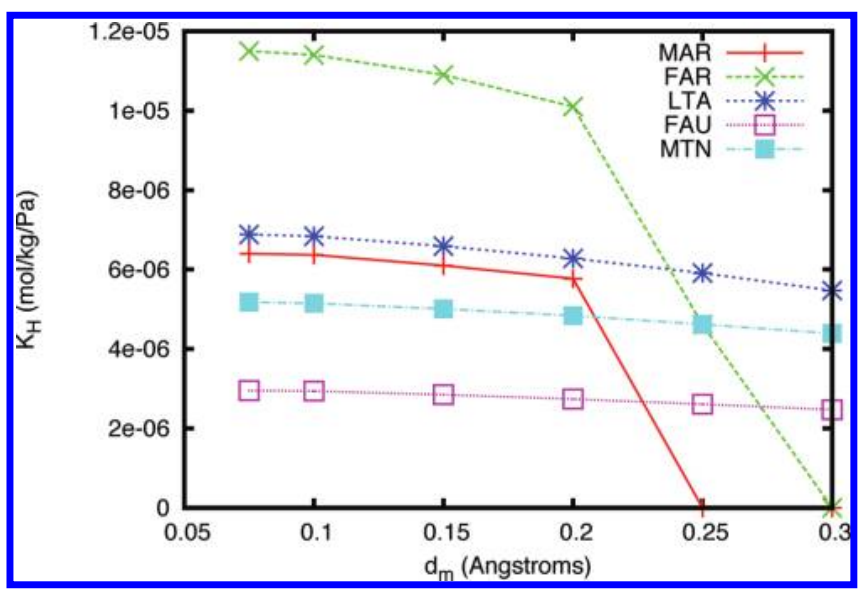

Figure 3. $\mathrm{CO}_{2} K_{\mathrm{H}}$ values for five IZA blocking set structures for mesh sizes of $d_{\mathrm{m}}=0.075,0.10,0.15,0.20,0.25$, and $0.30 \AA$. The sudden jump in the Henry coefficient values for zeolites MAR and FAR at $d_{\mathrm{m}}$ $=0.25$ and $0.30 \AA$ signify the effect of erroneous results due to insufficient accuracy of the energy grid.

seen from Figure 3, the $K_{\mathrm{H}}$ values decrease overall upon increasing $d_{\mathrm{m}}$. Most of the $K_{\mathrm{H}}$ contributions in the porous materials come from small regions with relatively low energy values due to the exponential Boltzmann term found in the formulation of the Henry coefficient in eq 1 . Because we use linear interpolation functions, it is not possible for the interpolated values to have energy values lower than the sampled grid points (as opposed to another form of interpolating functions, such as cubic splines, where it is possible for interpolated values to be lower). Thus, for smaller $d_{\mathrm{m}}$, the likelihood of sampling lower energy points increases due to general positive concavity of the energy profile around the local energy minimum regions. The choice of the linear interpolation functions was largely made to reduce the time spent in reading and interpolating the energy grid values. As can be seen from Figure 3, for small grid size, the $K_{\mathrm{H}}$ values do not change much as the percentage difference between $K_{\mathrm{H}}$ at $d_{\mathrm{m}}$ $=0.075 \AA$ and at $0.1 \AA$ is $<1 \%$. For grid sizes smaller than $d_{\mathrm{m}}=$ $0.075 \AA$, many of the IZA structures suffer from GPU memory allocation errors, since the device is bounded by the $3 \mathrm{~GB}$ DRAM. For zeolites MAR and FAR, the mesh size makes a significant difference, because, at lower grid resolutions of $d_{\mathrm{m}}=$ $0.25 \AA$, these structures are errorneously considered to be entirely inaccessible, reducing their $K_{\mathrm{H}}$ values to nearly zero. The sudden increase in the deviation for these structures demonstrates the importance of setting $d_{\mathrm{m}}$ sufficiently small to avoid inaccurate results. For experimentally known structures, the simulation $K_{\mathrm{H}}$ values can be compared to values derived from the experimental adsorption isotherm data at very low pressure values.

3.1.3. Performance: Energy Grid Size. Figure 4 summarizes the computational wall times for $20 \mathrm{CO}_{2} K_{\mathrm{H}}$ and $\Delta h_{i}$ calculations for different values of $d_{\mathrm{m}}$. As can be seen from Figure 4 , the total wall time $\left(T_{\text {tot }}\right)$ decreases significantly upon changing the grid size. At $d_{\mathrm{m}}=0.10,0.15,0.20,0.25$, and 0.30 $\AA$, $T_{\text {tot }}=396.82,117.93,55.37,32.67$, and $22.4 \mathrm{~s}$, respectively. There is a wall time improvement by a factor of 7.17 upon increasing $d_{\mathrm{m}}$ by 2 -fold, going from $0.10 \AA$ to $0.20 \AA$, which is a reasonable number, given that the number of grid points reduces by 8 -fold, affecting the energy grid, test rotation, and the pocket blocking routine. For the most part, the wall time for the Widom insertion routine is independent of the grid size, 


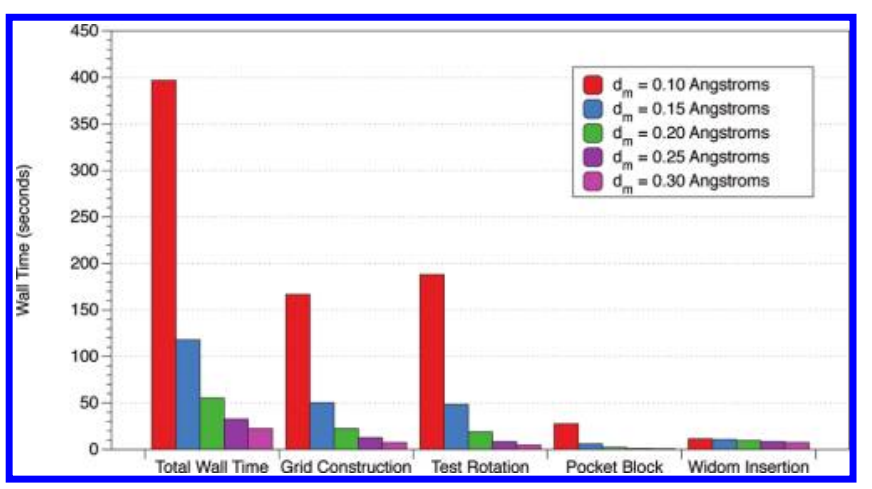

Figure 4. $\mathrm{CO}_{2} K_{\mathrm{H}}$ computational wall time for 20 IZA zeolite structures, as a function of energy grid mesh size. The computational intensity scales $\mathrm{O}\left(n^{3}\right)$ for all routines except for the Widom insertion, where $n$ is the number of energy grid points (inversely related to mesh size).

which is reflected in Figure 5, which shows the proportional wall time spent in each of the routines for different $d_{\mathrm{m}}$.

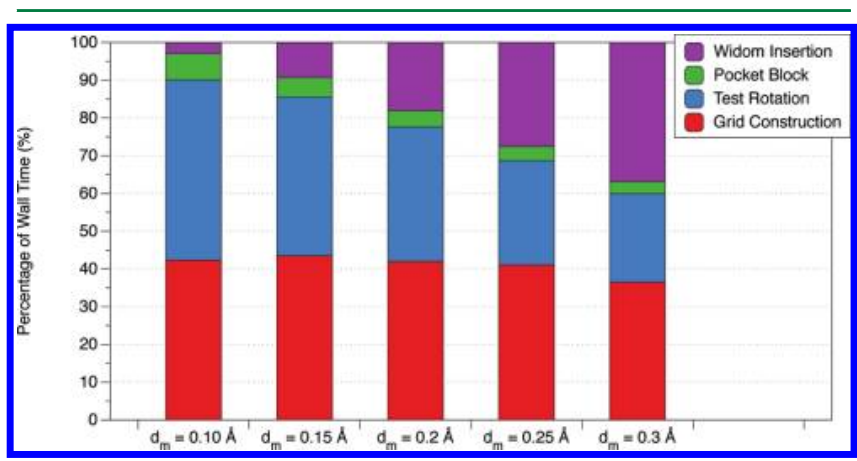

Figure 5. Proportional wall time spent in each of the main simulation routines for $20 \mathrm{CO}_{2} K_{\mathrm{H}}$ calculations at different grid size. From top to bottom are Widom insertion routine, pocket blocking routine, test rotation routine, and energy grid construction routine.

According to Figure 5, the proportional wall time spent in the Widom insertion routine increases for larger $d_{\mathrm{m}}$ values, while its absolute time remains relatively the same $(11.4,10.7,9.59,8.44$, and $7.55 \mathrm{~s}$ for $d_{\mathrm{m}}=0.10,0.15,0.20,0.25$, and $0.30 \AA$ ). From Figure 5, it can also be seen that the proportional wall time for the test rotation and the pocket blocking each reduce much more significantly (by factors of 42.4 and 39.2, respectively) when going from $d_{\mathrm{m}}=0.10 \AA$ to $d_{\mathrm{m}}=0.30 \AA$, compared to the energy grid routine (decrease by a factor of 22.3), despite the same reduction in the number of energy grid points in all three routines. It turns out that, in the energy grid construction routine, there exists separate computational terms within the Ewald summation that do not scale with the number of grid points and, therefore, the speedup improvement is smaller here, compared to the other two routines. Not shown in this work are the performance results for $\mathrm{CH}_{4}$, where similar speedup numbers are obtained by increasing $d_{\mathrm{m}}$. However, given that the test rotation routine is not called in the $\mathrm{CH}_{4}$ calculations, the proportional time spent in the Widom insertions is much larger for $\mathrm{CH}_{4}$, compared to the $\mathrm{CO}_{2}$ calculations for all $d_{\mathrm{m}}$ (but especially at large $d_{\mathrm{m}}$ ).

3.1.4. Performance: Number of Test Rotations $\left(N_{\text {rot }}\right)$. Next, we analyze the effect of changing the number of test rotations $\left(N_{\text {rot }}\right)$. As mentioned earlier, point particles such as $\mathrm{CH}_{4}$ do not require rotation moves and, therefore, are omitted in this analysis. We separately analyze the $\mathrm{CO}_{2} K_{\mathrm{H}}$ performance for the 20-member blocking set and the remaining 167-member nonblocking set (i.e., zeolites in which all regions are inaccessible) for IZA structures at $d_{\mathrm{m}}=0.10 \AA$ A. Figures 6a and $6 \mathrm{~b}$ show the computational wall times as a function of $N_{\text {rot }}$ for the two sets, respectively. In both sets, the qualitative behavior of the curves remains the same: the wall time starts high at small $N_{\text {rot }}$ decreases for larger $N_{\text {rot }}$ until a minimum wall time $\left(T_{\text {tot }}=328.96 \mathrm{~s}\right.$ for the blocking set and $T_{\text {tot }}=1270.43 \mathrm{~s}$ for the nonblocking set) is reached, and then increases monotonically for even larger $N_{\text {rot }}$. The minimum wall times, located at $N_{\text {rot }}=40$ in both sets, constitute a reduction of $17.1 \%$ and $26.6 \%$ from the default value of $N_{\text {rot }}=100$ rotations. The general behavior of the two curves can be explained by observing the changes in the two routines affected by the number of test rotations: (a) pocket blocking and (b) test rotation routines. For small $N_{\text {rot }}$, because of the small number of terms that inaccurately captures the true total energy landscape, the energy profile becomes less smooth and results in more disconnected regions inside the simulation box and more pockets. Accordingly, the pocket blocking routine spends more time in the flood fill algorithm and blocking pockets, resulting in longer wall time for the routine at small $N_{\text {rot }}$. For larger $N_{\text {rot }}$ the energy profile becomes smoother and less time is spent in the flood fill algorithm. Accordingly, the overall pocket blocking routine decreases monotonically with respect to $N_{\text {rot }}$. By contrast, the wall time for test rotation predictably scales linearly, with respect to $N_{\text {rot }}$ and the different behaviors

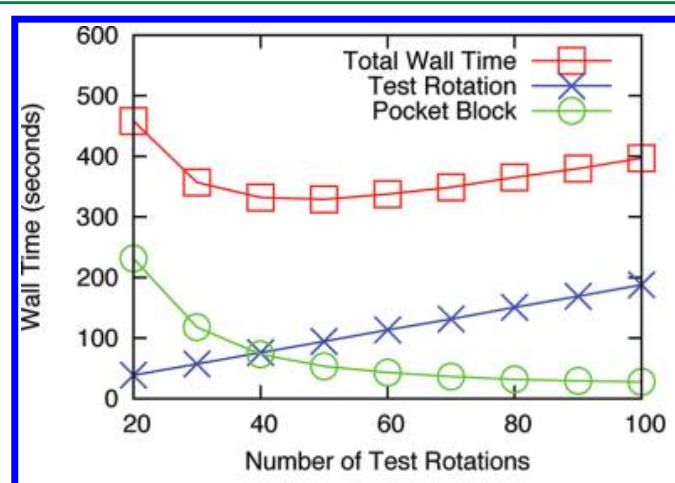

(a)

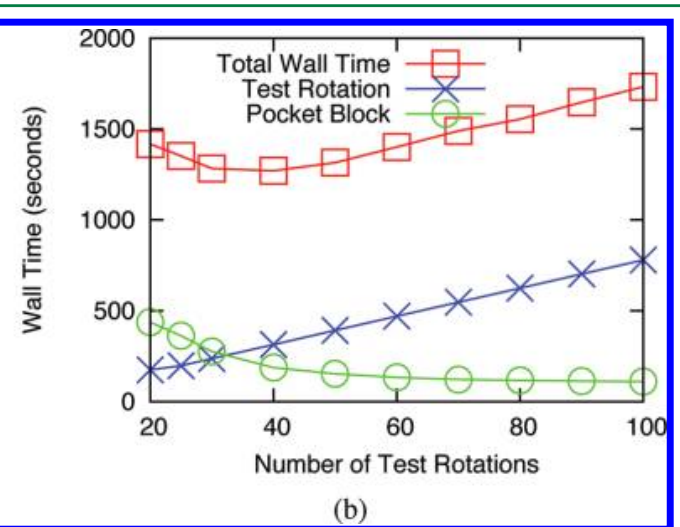

(b)

Figure 6. Computational wall time as a function of number of test rotations $\left(N_{\text {rot }}\right)$ for the $\mathrm{CO}_{2} K_{\mathrm{H}}$ calculations for the (a) 20-IZA blocking set and (b) the 167-IZA nonblocking set. The optimal number of test rotations that minimizes wall time occurs at $N_{\text {rot }}=40$. 
of these two routines contribute to the overall shape of the total wall time curves depicted in Figures $6 \mathrm{a}$ and $6 \mathrm{~b}$. In addition, there are small differences between the performance of the blocking set and that of the nonblocking set. In general, the proportional wall time spent in finding and detecting pockets is smaller in the nonblocking set compared to the blocking set; while the flood fill step is performed in both cases, only the blocking set is found to have regions that require blocking. Subsequently, one observes a steeper descent in the wall time curve at smaller numbers of test rotations in Figure 6a, compared to Figure $6 \mathrm{~b}$, because pocket blocking contributes relatively little to the proportional wall time for the nonblocking set.

At the optimal value of $N_{\text {rot }}=40$, the $K_{\mathrm{H}}$ results agree very well (within $0.1 \%$ ) from results obtained at $N_{\text {rot }}=100$ for both blocking and nonblocking sets that have relatively high $K_{\mathrm{H}}$. For zeolite structures that can be characterized by very low $K_{\mathrm{H}}$ values (i.e., $K_{\mathrm{H}}<10^{-18}$ ), the relative $K_{\mathrm{H}}$ difference between $N_{\text {rot }}$ $=40$ and $N_{\text {rot }}=100$ becomes large, but the difference here is uninteresting and ultimately meaningless in practice, because very small values of $K_{\mathrm{H}}$ all indicate poor adsorption properties of $\mathrm{CO}_{2}$, regardless of the exact number. Thus, there can be a performance gain by setting $N_{\text {rot }}=40$, from the default value of 100 , without having to sacrifice meaningful accuracy in the $K_{\mathrm{H}}$ results.

3.1.5. Performance: Pocket Blocking. Next, we discuss the performance details of the pocket blocking routine as a function of the number of CPU cores. Because the pocket blocking is the only routine of this algorithm that takes place entirely in the CPU, we utilize Pthreads to generate multiple CPU threads that work in parallel to accelerate the routine. Based on the profiling reported in ref 39, larger numbers of CPU threads are found to be generally advantageous for larger or more-complex zeolite structures, but there exists performance degradation in smaller or more-simplistic structures. The relationship between the number of CPU threads and the pocket blocking wall time is illustrated in Figure 7. As expected, the wall time decreases

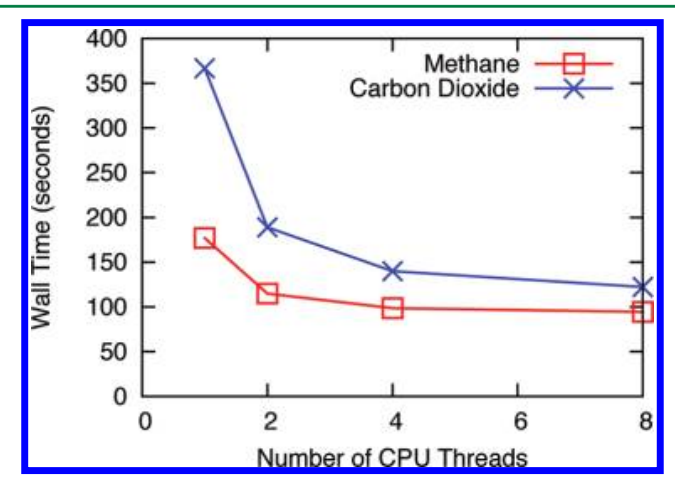

Figure 7. $\mathrm{CH}_{4}$ and $\mathrm{CO}_{2}$ pocket block routine wall time, as a function of the number of CPU threads, for the 187 IZA zeolite structures. The performance gain begins to saturate when going from 4 CPU threads to $8 \mathrm{CPU}$ threads.

monotonically, with respect to the number of CPU threads, for both the $\mathrm{CH}_{4}$ and the $\mathrm{CO}_{2}$ simulations and the saturation point is reached near 4-8 CPU threads. For $\mathrm{CO}_{2}$ simulations, the performance scales almost linearly; going from 1 to $2 \mathrm{CPU}$ threads a speedup by a factor of 1.96 is observed (in $\mathrm{CH}_{4}$, a speedup by a factor of 1.53 is observed).

3.1.6. Performance: GPU vs CPU. Finally, we assess performance differences between the CPU and the GPU. For the $\mathrm{CH}_{4}$ energy grid routine, we observe speedups by factors of $\sim 50$, compared to an optimized single CPU core simulation without SSE from simulation results from the Carver cluster at NERSC. The number is reasonable, given that the energy grid construction is compute-bound, because of its high computational intensity and most of the memory transactions are handled inside the fast constant memory of the GPU. We have not written a CPU version of the code for the $\mathrm{CO}_{2}$ energy grid routine, but we expect the speedup numbers to be similar, given the similar computational flop intensity and similar memory references and coalescing patterns. Moreover, the inclusion of a fast pocket blocking routine accelerates the detection of inaccessible regions, compared to slow methods that are based on molecular dynamics.

3.2. Characterization of Zeolites. Utilizing the total energy grid, we can create another grid (i.e., local $K_{\mathrm{H}}$ grid) that contains the Boltzmann factors at each of the points. The local $K_{\mathrm{H}}$ grid can be used to attain information about the locations likely to be occupied by the gas molecules, providing another means to characterize the structures in the post-processing stage after the simulation. In Figure 8, we include an example illustration that displays the effect of pocket blocking in the zeolite LTA structure. A heat mapping represents the local $\mathrm{CO}_{2}$ $K_{\mathrm{H}}$ regions, with warmer colors denoting larger $K_{\mathrm{H}}$ contributions, and as can be seen, the distribution of the local $K_{\mathrm{H}}$ changes upon enabling/disabling the pocket blocking routine. Specifically in the case of LTA, the corner regions in the unit cell become inaccessible as $\mathrm{CO}_{2}$ cannot enter into this region within a reasonable experimental time scale. Accordingly, the $\mathrm{CO}_{2} K_{\mathrm{H}}$ values inside LTA with or without pocket blocking are $9.59 \times 10^{-6}$ and $6.85 \times 10^{-6} \mathrm{~mol} \mathrm{~kg}^{-1} \mathrm{~Pa}^{-1}$, as the $K_{\mathrm{H}}$ value decreases upon setting the energy grid values inside the inaccessible region to be very high. In general, structures that contain inaccessible regions always show a reduction in $K_{\mathrm{H}}$ values as proper inclusion of the pocket blocking raises the energy values in these regions. We illustrate by this example the importance of pocket blocking in avoiding the overestimation of the level of adsorption in a given porous material.

Until now, the analysis has been focused on the experimentally verified IZA structures, which comprise a very small subset of the entire database of zeolite structures. The algorithm explained earlier can be utilized unchanged to easily extend our simulation code to process 135224 hypothetical zeolites in the database. Figure 9 plots the histogram of both the $\mathrm{CO}_{2}$ and $\mathrm{CH}_{4} K_{\mathrm{H}}$ values for all of the zeolite structures. Given that zeolites are seen as one of the ideal candidates for carbon capture, it is not surprising that the $K_{\mathrm{H}}$ values for $\mathrm{CO}_{2}$ are generally higher than that of $\mathrm{CH}_{4}$. The broader distributions of the $\mathrm{CO}_{2} K_{\mathrm{H}}$ values also indicate that the range of possible structures with different $\mathrm{CO}_{2}$ adsorption properties remains large, compared to $\mathrm{CH}_{4}$. Extrapolating from the simulation times obtained from the IZA structures, we can obtain the $\mathrm{CO}_{2}$ $K_{\mathrm{H}}$ values of the entire hypothetical zeolite structures in $\sim 50 \mathrm{~h}$ of total wall time, utilizing 8 Tesla C2050 GPUs in the Dirac cluster. At the end of the $K_{\mathrm{H}}$ calculations, selected structures from a large database that are deemed to have shown good adsorption properties can be analyzed in-depth by utilizing the GPU GCMC simulations whose algorithm is described in Section 2.4. The predicted adsorption properties obtained from the simulation code can provide valuable insights to experimentalists interested in synthesizing materials inside a large porous materials database. 


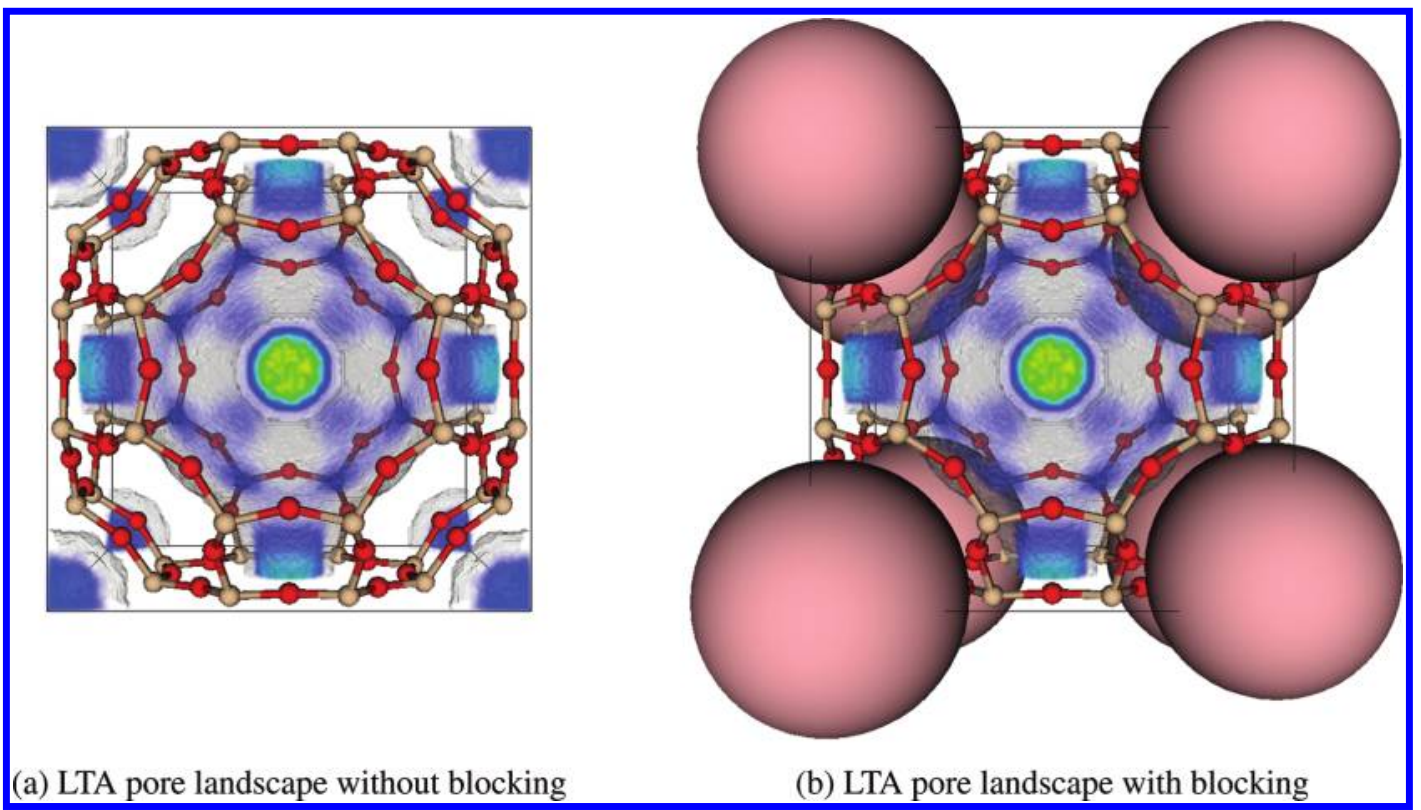

Figure 8. Snapshots of LTA zeolite with pore landscape (a) without blocking and (b) with blocking. The silicon and oxygen atoms of the framework are shown in tan and red, respectively. The local Henry coefficient contributions are shown as a heat map; warmer colors indicate higher likelihood of the guest $\mathrm{CO}_{2}$ molecule occupying these positions. The inaccessible pocket region is shown to be fully excluded by the pink spheres, located on the eight corners of the unit cell. Upon enabling the pocket blocking routine, the energy grid points in the blocking region are set to very high energy and the $\mathrm{CO}_{2}$ molecules are forced away from these regions.

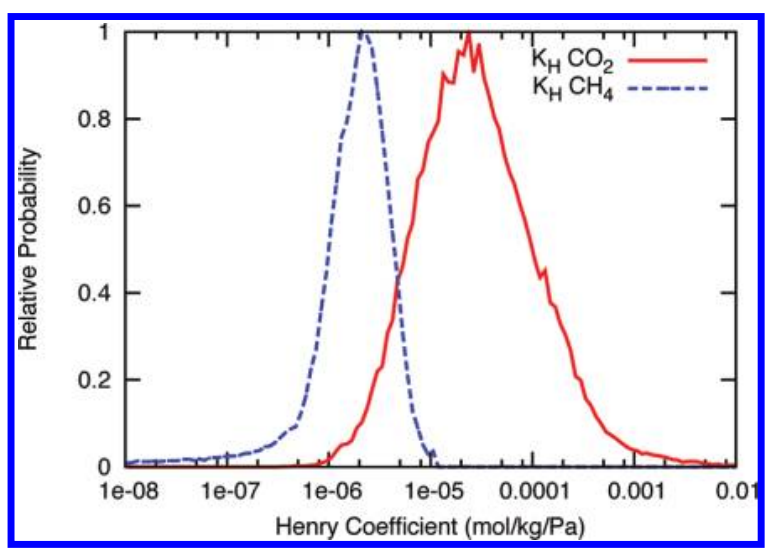

Figure 9. Distribution of $\mathrm{CO}_{2}$ and $\mathrm{CH}_{4}$ Henry coefficient values for 135224 hypothetical zeolite structures. Generally, the $\mathrm{CO}_{2} K_{\mathrm{H}}$ values are larger than the $\mathrm{CH}_{4} K_{\mathrm{H}}$, indicating that zeolites have high selectivity for $\mathrm{CO}_{2}$.

\section{SUMMARY AND FUTURE WORK}

We have developed a graphics processing unit (GPU)-based simulation code that can characterize and prescreen a large database of porous materials. Our code has the capability to quickly compute the Henry coefficient and the heats of adsorption values for many different gas molecules immersed inside a porous material. We can further analyze individual structures in the simulations by visualizing local Henry coefficient values to determine local adsorption properties of a given material. Although the simulation results presented in this work pertain to zeolites, the code can be easily extended to process other classes of microporous materials. For future work, we plan to (a) analyze the effect of adding cations to zeolites and (b) characterize a large database of other porous materials, such as MOF and ZIF structures.

\section{AUTHOR INFORMATION}

\section{Corresponding Author}

*E-mail: jihankim@lbl.gov.

\section{Notes}

The authors declare no competing financial interest.

\section{ACKNOWLEDGMENTS}

This research used resources of the National Energy Research Scientific Computing Center, which is supported by the Office of Science of the U.S. Department of Energy under Contract No. DE-AC02-05CH11231. J.K., R.L.M., O.R., and M.H. were supported by the Director, Office of Science, Advanced Scientific Computing Research, of the U.S. Department of Energy under Contract No. DE-AC02-05CH11231. In addition, R.L.M. and O.R. were supported by the Scientific Discovery through Advanced Computing (SciDAC) program's Visualization and Analytics Center for Enabling Technologies (VACET). M.H. was supported jointly by DOE Office of Basic Energy Sciences and the Office of Advanced Scientific Computing Research, through SciDAC Project No. CSNEW918 entitled "Knowledge guided screening tools for identification of porous materials for $\mathrm{CO}_{2}$ separations". B.S. was supported as part of the Center for Gas Separations Relevant to Clean Energy Technologies, an Energy Frontier Research Center funded by the U.S. Department of Energy, Office of Science, Office of Basic Energy Sciences under Award No. DESC0001015.

\section{REFERENCES}

(1) Auerbach, S. M.; Carrado, K. A.; Dutta, P. K. Handbook of Zeolite Science and Technology; Marcel Dekker: New York, 2004.

(2) Smit, B.; Maesen, T. L. M. Nature 2008, 457, 671-677.

(3) Smit, B.; Maesen, T. L. M. Chem. Rev. 2008, 108, 4125-4184.

(4) Krishna, R; van Baten, J. M. Chem. Eng. J. 2007, 133, 121-131.

(5) Haldoupis, E.; Nair, S.; Sholl, D. S. J. Am. Chem. Soc. 2010, 132, $7528-7539$. 
(6) Haldoupis, E.; Nair, S.; Sholl, D. S. Phys. Chem. Chem. Phys. 2011, 13, 5053-5060.

(7) Lin, L.-C.; Berger, A.; Martin, R. L.; Kim, J.; Swisher, J.; Jariwala, K.; Rycroft, C. H.; Bhown, A.; Deem, M. W.; Haranczyk, M.; Smit, B. Nat. Mater., accepted.

(8) D'Alessandro, D. M.; Smit, B.; Long, J. R. Angew. Chem., Int. Ed. 2010, 49, 6058-6082.

(9) Millward, A. R.; Yaghi, O. M. J. Am. Chem. Soc. 2005, 127, 17998-17999.

(10) Walton, K. S.; Millward, A. R.; Dubbeldam, D.; Frost, H.; Low, J. J.; Yaghi, O. M.; Snurr, R. Q. J. Am. Chem. Soc. 2008, 130, 406-407.

(11) Banerjee, R.; Phan, A.; Wang, B.; Knobler, C.; Furukawa, H.; O'Keeffe, M.; Yaghi, O. M. Science 2008, 319, 939-943.

(12) Sumida, K.; Hill, M. R.; Horike, S.; Dailly, A.; Long, J. R. J. Am. Chem. Soc. 2009, 131, 15120-15121.

(13) Choi, H. J.; Dinca, M.; Long, J. R. J. Am. Chem. Soc. 2008, 130, $7848-7850$.

(14) (a) Baerlocher, C.; Meier, W. M.; Olson, D. H. Atlas of Zeolite Framework Types, Seventh Ed.; Elsevier: Amsterdam, 2007. (b) http://www.iza-online.org/ (accessed Jan 1, 2010).

(15) (a) Lach-hab, M.; Yang, S.; Vaisman, I. I.; Blaisten-Barojas, E. Mol. Inf. 2010, 29, 297-301. (b) Carr, D. A.; Lach-hab, M.; Yang, S.; Vaisman, I. I.; Blaisten-Barojas, E. Microporous Mesoporous Mater. 2009, 117, 339-349.

(16) Foster, M. D.; Treacy, M. M. J. http://www. hypotheticalzeolites.net (accessed Nov 13, 2009)

(17) Earl, D. J.; Deem, M. W. Ind. Eng. Chem. Res. 2006, 45, 54495454.

(18) Deem, M. W.; Pophale, R.; Cheeseman, P. A.; Earl, D. J. J. Phys. Chem. C 2009, 113, 21353-21360.

(19) Pophale, R.; Cheeseman, P. A.; Deem, M. W. Phys. Chem. Chem. Phys. 2001, 13, 12407-12412.

(20) Maginn, E. J.; Bell, A. T.; Theodorou, D. N. J. Phys. Chem. 1995, 99, 2057-2079.

(21) Macedonia, M. D.; Maginn, E. J. Mol. Phys. 1999, 96, 13751390.

(22) Smit, B.; Krishna, R. Curr. Opin. Solid State Mater. Sci. 2001, 5, 455-461.

(23) Willems, T. F.; Rycroft, C. H.; Kazi, M.; Meza, J. C.; Haranczyk, M. Microporous Mesoporous Mater. 2012, 149, 134-141.

(24) Wei, J.; Floudas, C. A.; Gounaris, C. E.; Somorjai, G. A. Catal. Lett. 2009, 133, 234-241.

(25) Foster, M. D.; Rivin, I.; Treacy, M. M. J.; Friedrichs, O. D. Microporous Mesoporous Mater. 2006, 90, 32-38.

(26) Martin, R. L.; Smit, B.; Haranczyk, M. J. Chem. Inf. Model. 2012, $52,308-318$.

(27) Owens, J. D.; Luebke, D.; Govindaraju, H.; Harris, M; Krüger, J; Lefohn, A. E.; Purcell, T. Comput. Graph. Forum 2007, 26 (1), 80113.

(28) Kim, J.; Rodgers, J.; Athènes, M.; Smit, B. J. Chem. Theory Comput. 2011, 7, 3208-3222.

(29) Preis, T.; Virnau, P.; Schneider, J. J. Comput. Phys. 2009, 228, 4468-4477.

(30) Li, H.; Petzold, L. Int. J. High Perform. Comput. 2010, 24, 107116.

(31) Anderson, A.; Goddard, W.; Schroder, P. Comput. Phys. Commun. 2007, 177, 298-306.

(32) Garcia-Perez, E.; Parra, J. B.; Ania, C. O.; Garcia-Sanchez, A.; van Baten, J. M.; Krishna, R.; Dubbeldam, D.; Calero, S. Adsorption 2007, 13, 469-476.

(33) Dubbeldam, D.; Calero, S.; Vlugt, T.; Krishna, R.; Maesen, T.; Beerdsen, E.; Smit, B. Phys. Rev. Lett. 2004, 93, 088302.

(34) (a) Dubbeldam, D.; Smit, B. J. Phys. Chem. B 2003, 107, 12138.

(b) Bates, S. P.; v. Well, W. J. M.; v. Santen, R. A.; Smit, B. J. Am. Chem. Soc. 1996, 118, 6753.

(35) Krishna, R.; van Baten, J. M. Langmuir 2010, 26, 2975-2978.

(36) Keffer, D.; Gupta, V.; Kim, D.; Lenz, E.; Davis, H. T.; McCormick, A. V. J. Mol. Graph. 1996, 14, 108-116.
(37) Haranczyk, M.; Sethian, J. A. J. Chem. Theory Comput. 2010, 6, $3472-3480$.

(38) Haranczyk, M.; Sethian, J. A. Proc. Natl. Acad. Sci., U.S.A. 2009, 106, 21472-21477.

(39) Martin, R. L.; Prabhat; Donofrio, D.; Sethian, J. A.; Haranczyk, M. J. High Perform. Comput. Appl. in press, DOI: 10.1177/ 1094342011431591.

(40) Toukmaji, A. Y.; Board, J. A. Comput. Phys. Commun. 1996, 95, 73-92. 